\title{
Los artistas colombianos y las plataformas de música digitales: algunas dificultades*
}

\section{Marcela Palacio Puerta**}

Resumen: Internet brinda nuevas oportunidades de negocios legales para la industria musical y en especial para artistas o discográficas independientes. Lo anterior se debe a la gran proliferación y crecimiento de plataformas de música digitales. No obstante, contrario a las estadísticas, los artistas no han podido beneficiarse de la manera esperada de dichas oportunidades. El desarrollo académico de este tema se encuentra en sus inicios, especialmente respecto del panorama colombiano, así que, por primera vez en la literatura, se busca abarcar algunas dificultades a las que se enfrentan los artistas colombianos en el mundo de la música digital.

Palabras clave: Industria musical, Colombia, plataformas digitales, derechos conexos, agregadores, regalías.

\section{Colombian Artists and Digital Music Platforms: Some Difficulties}

Aвstract: The Internet provides new business opportunities for the music industry, especially for both independent artists and record companies. The reason

Fecha de recepción: 24 de marzo de 20I7. Fecha de aceptación: 9 de octubre de 20I7. Para citar este artículo: Palacio Puerta, M. "Los artistas colombianos y las plataformas de música digitales: algunas dificultades", Revista de Derecho Privado, Universidad Externado de Colombia, n. ${ }^{\circ} 33$, julio-diciembre de 201 7, I I I-I33. DOI: https://doi.org/Io. I 860I/or 234366.n33.05

* Doctora en derecho, American University Washington College of Law. Docente investigadora, Universidad Sergio Arboleda, Bogotá, Colombia. Contacto: marcelapalaciop@gmail.com. La autora agradece a María Camila Carrillo por su valiosa colaboración en esta investigación. 
of the latter is the great proliferation and growth of digital music platforms. However, contrary to statistics, artists have not been able to benefit of such opportunities in the expected manner. The academic development on this subject is in its beginnings especially with respect to the Colombian panorama, therefore for the first time in the literature, this paper draws some of the difficulties that the Colombian artists face in the world of the digital music.

Keywords: Music industry, Colombia, digital platforms, neighboring rights, aggregators, royalties.

\section{Introducción}

Los artistas y en general todos los que viven del derecho de autor dependen en un alto porcentaje de las regalías que genera el autorizar el uso de sus obras. Este es el caso de los músicos, tanto los compositores como los intérpretes y ejecutantes, roles que, aunque algunas veces coinciden en las mismas personas, no siempre sucede así. Este artículo se enfoca en la situación de los segundos.

Anteriormente, para que un artista pudiera distribuir o comercializar su música, la mejor opción, o tal vez la única opción, era firmar un contrato con un gran sello discográfico como Sony Music Entertainment o Universal Music Group. Hoy en día, gracias al auge y el desarrollo de internet, se han presentado nuevas oportunidades para los artistas, especialmente para los independientes.

Lo anterior se debe a que internet brinda el escenario ideal con alcance mundial para que sean los mismos artistas los que promocionen su música, se den a conocer en el mercado, y a su vez obtengan y recolecten las regalías por el uso. Esto se ha logrado debido a la gran acogida que han tenido las diferentes plataformas digitales de música que ofrecen contenido legal mediante diferentes modelos comerciales, a diferencia de los años noventa cuando predominaba el uso de las alternativas piratas para el consumo musical, tales como Ares, Kazaa o Napster. No obstante, a pesar de ser este un gran avance para los artistas, no todo es color rosa.

Actualmente, el problema para los artistas musicales alrededor del mundo que buscan explotar sus creaciones en el espacio digital ha consistido en la falta de transparencia por parte de la industria de las plataformas de música digitales y demás intervinientes en el momento de rendir cuentas sobre las regalías recolectadas y su repartición entre los distintos intervinientes de la cadena de valor. De manera adicional, para los artistas colombianos, el mundo de las plataformas de música digitales ha presentado otras complicaciones, como la dificultad de saber cuáles regalías se están recolectando y tener que someterse a jurisdicciones extranjeras.

A pesar de ser un tema de alto impacto para la industria musical, la literatura existente acerca del modelo de negocios de música digital y su impacto para los 
artistas es limitada, y mucho más cuando se enfoca a la situación colombiana. Por consiguiente, esta investigación se convierte en el primer escalón para el tratamiento del tema.

\section{El nuevo panorama que ofrece internet: oportunidad para la distribución y comercialización}

A finales de los años noventa y principios del año dos mil parecía que internet se estaba convirtiendo en el enemigo de la industria musical. La proliferación de sistemas peer-to-peer que permitían descargar música infringiendo los derechos de autor tenía preocupada a la industria. Abundaron en las cortes del mundo, en especial las estadounidenses, los casos contra este tipo de modelos de negocios infractores como Napster, Grokster, Kazaa, entre otros ${ }^{\mathrm{I}}$. De igual manera, la industria musical hacía lobby en búsqueda de expandir la protección del derecho de autor y en especial para obtener herramientas de protección en este nuevo ambiente en crecimiento conocido como internet. Fue así como aparecieron, por ejemplo, normas como Digital Millennium Copyright Act en r 998 en Estados Unidos, la cual buscaba brindar protección a los creadores en el ambiente digital para impulsar su uso como medio de explotación de obras protegidas ${ }^{2}$.

No obstante, hoy en día con el avance de las tecnologías se empieza a observar un cambio en la relación del uso de internet con la industria de la música. Internet se convierte en un escenario lleno de posibilidades tanto para la creación como para la distribución, marketing y recolección de regalías de los músicos ${ }^{3}$. Artistas alrededor del mundo comienzan a utilizar internet como herramienta para darse a conocer, ganar y recolectar regalías, sin tener la necesidad de disponer de un contrato firmado con una discográfica. Es decir, se vuelve la herramienta ideal para músicos independientes.

Este sería el caso del famoso cantante canadiense Justin Bieber o del británico James Bay. Respecto de Justin Bieber, se dio a conocer en 2008 gracias a videos subidos por su madre a la plataforma digital YouTube, que obtuvieron millones de vistas, donde el adolescente interpretaba a diversos artistas. Esta situación llamó la atención del manager Scooter Braun, quien llevó a Justin inicialmente a firmar un contrato con el sello Raymond Braun Media Group y

I Ríos, W. "Análisis de las sentencias en los casos de Napster, Grokster, Morpheus, Streamcast y Kazaa”, La Propiedad Inmaterial, I 2, 2008, 59, 86. Recuperado de https://dialnet.unirioja.es/ servlet/articulo? codigo $=3$ I 35280

2 Balaban, D. "The Battle of the Music Industry: the Distribution of Audio and Video Works via the Internet, Music and More", Fordham Intellectual Property Media and Entertainment Law Fournal, I 2, 2002, 258 .

3 Carrier, M. "No, riaA, It's not the End of the World for Musicians", University of MissouriKansas City Law Review, 2014, 287-392. Recuperado de https://papers.ssrn.com/sol3/papers. cfm?abstract_id=2602469 
un contrato de grabación con Islands Records ${ }^{4}$. Respecto del artista James Bay, quien se dedicaba a tocar en bares, se dio a conocer cuando algún miembro del público decidió subir una de sus interpretaciones a YouTube y la discográfica Universal Republic, de Nueva York, decidió contactarlo5.

El uso de internet como herramienta de promoción para los artistas musicales se debe al surgimiento de nuevos modelos de negocios legales que permiten tanto a artistas independientes como a sellos discográficos, ya sean independientes o grandes corporaciones, tomar ventaja de la red como un nuevo escenario para el desarrollo de negocios. El avance y proliferación de servicios de streaming online o de descargas como YouTube, YouTube Red, iTunes, Google Play, Amazon, Deezer, entre otras, brindan nuevas oportunidades para explotar las obras musicales ${ }^{6}$.

Dichas plataformas ofrecen diferentes tipos de servicios diseñados para diversos usuarios. Por ejemplo, plataformas como iTunes Music Store ofrecen el servicio de descarga, es decir, el usuario adquiere una copia de la canción, generalmente luego del pago de un precio. Dicho precio para Colombia está en aproximadamente $\$ 3,870$ (Us\$ I,29) por canción7. Por otro lado, plataformas como Spotify en su servicio Premium ofrecen un servicio de modelo interactivo en el cual el usuario puede escoger qué canción escuchar y adicionalmente crear una copia de dicha canción en su dispositivo que es accesible mientras se mantenga la suscripción mediante un pago mensual que varía según el país. En Colombia, por ejemplo, el servicio de Spotify Premium tiene un valor de \$I 4.900 pesos mensuales. Finalmente, existen modelos financiados por publicidad, donde el usuario a cambio de escuchar música debe ver o escuchar anuncios publicitarios; por ejemplo, la versión gratuita de Spotify, Pandora o YouTube ${ }^{8}$. A excepción de YouTube, generalmente estos servicios se caracterizan por no ser interactivos, es decir, el usuario no tiene la posibilidad de elegir la canción a escuchar.

Esta nueva industria de música digital permite a los artistas distribuir sus obras incluso a mercados a los que no accedían con anterioridad. Por ejemplo, la

4 "Justin Bieber: la biografía del cantante canadiense", La Prensa, 29 de octubre de 20r5. Recuperado de http://laprensa.peru.com/espectaculos/noticia-justin-bieber-biografia-539 5 ; ver también Blanca, "Justin Bieber. Biografía completa", Prensa corazón, s. f. Recuperado de http:// laprensa.peru.com/espectaculos/noticia-justin-bieber-biografia-539 5

5 Mclean, "James Bay interview: 'As a solo artist with a guitar, I can only write so many weepie little bedroom songs"”, Independent, s. f. Recuperado de http://www.independent.co.uk/artsentertainment/music/features/james-bay-interview-as-a-solo-artist-with-a-guitar-i-can-onlywrite-so-many-weepie-little-bedroom-IOI 2 I $453 \cdot \mathrm{html}$

6 Donald, A., "Independent Musicians: Let Technology Free you From Labels' Yoke", The Associated Press, enero 27, 2004.

7 Rethink Music. Fair Music (música justa): transparencia y flujos monetarios en la industria musical, Boston, Berklee ICE, s. f., 3 .

$8 \quad$ Ibid., 8. 
disquera británica independiente Beggars Group afirma que utilizando los servicios de streaming está presente en los mercados de Nigeria, Angola y Uganda por primera vez, y reconoce que estos nuevos mercados representan el veinte por ciento de los ingresos del grupo por streamings9.

Otra especial ventaja de los nuevos modelos de negocio digitales, además de permitirles a los artistas distribuir sus obras sin la necesidad de firmar con una disquera, es que al ser legales estas plataformas digitales generan ingresos para los artistas. Por ejemplo, en el caso de YouTube brinda la posibilidad a los artistas de obtener regalías por el uso de sus canciones, ya sea a través de un agregador -como se verá adelante- o a través de su herramienta Content ID, diseñada por YouTube buscando proteger los derechos de autor, especialmente de los titulares de obras musicales de gran acogida por el público y que son usualmente subidas a la plataforma por los usuarios. Dicha herramienta permite cotejar todos los videos que se suben a la plataforma con el archivo de la obra que previamente le proporcione el titular de derechos a YouTube. Una vez que se encuentra que un video subido por uno de los usuarios de la plataforma coincide con la obra de un titular miembro de Content ID, YouTube le permite al titular monetizar dicho video o tomar otras medidas, como silenciar el video o bajarlo de la plataforma ${ }^{\mathrm{IO}}$. De igual manera, otras plataformas también pagan regalías a los artistas por el uso de sus canciones. Por ejemplo, Spotify paga por los streamings que suceden en la plataforma y iTunes por cada una de las descargas.

Adicionalmente, los artistas se pueden ver motivados a utilizar el mercado de la música digital, dado su crecimiento. En los últimos años el mercado digital ha crecido a escala mundial y ha superado el mercado de las ventas análogas, volviéndose la corriente principal de ingresos de la música grabada. Del total de los ingresos de la industria de la música, el $45 \%$ proviene del uso digital frente a un $39 \%$ de ventas físicas ${ }^{11}$. Por ejemplo, en el caso de Colombia, en 2015 el $77,5 \%$ del ingreso que generó la música se encontraba relacionado con el mundo digital ${ }^{\mathrm{I} 2}$. De estos nuevos modelos de negocio digitales se destaca el crecimiento económico que ha tenido el uso del streaming tanto en su modelo gratuito como en el modelo de suscripción, superando el número de descargas en aproximadamente 42 países y representando el $43 \%$ de los ingresos de la industria digital a escala global ${ }^{13}$.

\footnotetext{
$9 \quad$ IFPI, Global Music Report: Music Consumption Exploding Worldwide, 2016, I9.

IO "Cómo funciona Content ID", YouTube, s. f. Recuperado de https://support.google.com/youtube/answer/2 797370 ?hl=es-4I 9

I I IFPI, Global Music Report, cit., 8 .

i 2 Guzmán, J. "La música por Internet le gana la batalla a los discos”, El Tiempo, mayo i 5, 20 i6. Recuperado de http://www.eltiempo.com/entretenimiento/musica-y-libros/ventas-de-musicadigital-sobrepasaron-las-de-discos/I6593353

I 3 IFPI, Global Music Report, cit., I 7 .
} 
De esta forma, se puede observar cómo la industria de la música digital ha tenido gran impacto económico en la industria y se ha convertido en una herramienta para que los artistas, y sobre todos aquellos que son independientes, logren distribuir, comercializar y recaudar regalías.

\section{Acceso al mercado y recaudación de regalías: los agregadores}

Aunque, como se mencionó anteriormente, las plataformas como Spotify, Deezer, iTunes, entre otras, son una excelente alternativa para músicos y discográficas independientes, ya que no hacen falta los contactos y el poder de comercialización y distribución que representa un sello discográfico grande ${ }^{\mathrm{I} 4}$ para poder acceder al mercado, dichas plataformas no permiten a los artistas poner a disposición sus composiciones o interpretaciones directamente en sus listas de reproducción. Una de las razones es que resulta dispendioso para las plataformas de música digital entrar a negociar licencias con cada artista independiente, o discográficas pequeñas o independientes. Igualmente, sería una labor complicada para la plataforma verificar la titularidad de las obras musicales ${ }^{15}$. Por lo tanto, dichas plataformas exigen a los mencionados artistas o discográficas acceder a sus servicios a través de un intermediario denominado agregador que se encargue de realizar dichas labores.

Esta situación es diferente para los músicos que han firmado contratos con una discográfica grande, como Sony Music Corporation, Universal Music Group o Warner Music Group, ya que en este caso será la discográfica quien tenga acceso directo a las plataformas digitales, tanto para poner a disposición su contenido como para negociar las tarifas y recolectar regalías. En este caso la suma que los artistas representados recibirán por la explotación de sus obras estará guiada por los términos de los contratos firmados con la discográfica ${ }^{16}$. La situación anterior se puede evidenciar en la página para artistas de Spotify, donde se establece: "Lo primero es lo primero. Poner tu música en Spotify es fácil. Si tú tienes una discográfica o agregador, ellos lo harán por ti. Si no tienes, nosotros tenemos acuerdos con diversas compañías que podrán entregarnos tu música y recaudar tus regalías" ${ }^{7}$.

I4 "Who Needs Record Labels?", Independent, marzo I 2, 2010. Recuperado de http://www.independent.co.uk/arts-entertainment/music/features/who-needs-record-labels- I $920056 . \mathrm{html}$

I5 Galuszka, P., "Music aggregators and Intermediation of the Digital Music Market", International Fournal of Communication, 9, 2015, 262-264.

I6 Rethink Music. Fair Music (música justa)..., cit., 4.

I7 Spotify. "Your Music", s. f. Recuperado de https://artists.spotify.com/guide ("First things first. Getting your music on Spotify is easy. If you have a label or aggregator, they'll handle the work for you. If you don't, we have deals in place with a number of companies who can deliver your music to us and collect royalties for you") (traducción no oficial). 
Los agregadores son empresas intermediarias entre los artistas y las plataformas digitales. Algunos ejemplos de agregadores son CD Baby, Tunecore, Record Union, Awa, Emubands. Desde el punto de vista de los artistas, los agregadores se encargan de distribuir las obras musicales en tiendas digitales, entre otros servicios, permitiendo tener una distribución global de la música, y a la vez se encargan de recolectar las regalías causadas por la explotación de las obras por streaming o descargas. Por ejemplo, TuneCore promociona sus servicios como "la mejor manera de vender tu música en iTunes, Amazon, Spotify y más [...] obtiene un trato justo. Mantiene el roo \% de los ingresos de ventas [...]" ${ }^{\prime} 8$.

Desde el punto de vista de las plataformas digitales de música, los agregadores se encargan de obtener y otorgarles las licencias necesarias sobre las obras musicales para poder ponerlas a disposición en las plataformas, especialmente frente al fonograma y derechos de los intérpretes y ejecutantes. Una característica principal de los agregadores es que celebran contratos con exclusividad con los artistas, es decir, un artista no puede contratar su repertorio con más de un agregador. No obstante, una plataforma digital sí puede celebrar contratos con diversos agregadores, y los agregadores podrán distribuir su música con diversas plataformas $^{19}$.

Las regalías por explotación que los agregadores recauden van a depender del tipo de servicio adquirido por parte del artista. Por ejemplo, algunos agregadores ofrecen servicios de music distribution para el recaudo de regalías relativo a los derechos del productor fonográfico por las diferentes ventas y reproducciones del fonograma. Este servicio lo ofrecen agregadores como TuneCore ${ }^{20}$. Otro tipo de servicio que los artistas pueden adquirir se refiere al publishing management, es decir, para recaudar derechos de autor sobre las composiciones para recolectar por reproducciones en streaming y en descargas y por comunicación pública, entre otros tipos de regalías; este servicio lo ofrecen agregadores como CD Baby ${ }^{2}$.

No obstante, la intermediación de los agregadores para los servicios anteriormente mencionados, y otros más, no es gratuita; el valor de la comisión varía si se trata de artistas individuales o de discográficas, dado el tamaño del catálogo. Una discográfica con un catálogo considerable podría conseguir un precio más bajo con un agregador ${ }^{22}$. En el caso de artistas individuales, el valor por el servicio de intermediación y el porcentaje de regalías recibido por los artistas varía

I8 TuneCore, TuneCore, s. f. Recuperado de http://www.tunecore.com/

I9 Galuszka, P., "Music aggregators and Intermediation of the Digital Music Market", cit., 262.

20 TuneCore, "What is the difference between Music Distribution and Publishing administration? Do I need Publishing Administration?”, enero 26 de 20r6. Recuperado de http://pub. help.tunecore.com/app/answers/detail/a_id/255

2 I cDbaby, "Make more money with music publishing", s. f. Recuperado de https://members.cdbaby.com/pro.aspx

22 Galuszka, P., "Music aggregators and Intermediation of the Digital Music Market", cit., 263. 
dependiendo de cada agregador. Por ejemplo, Emubands paga a los artistas el ıо० \% de las regalías obtenidas por la venta de la música y el precio del servicio se basa en una tasa única de acuerdo con el número de canciones publicadas y por publicación. Así, de I a 2 canciones tendrán un precio de Us $\$ 42,50$; de 3 a 5 canciones, us $\$ 59,95$; de 6 a 20 canciones, us $\$ 84,95^{[23]}$, mientras que Record Union, otro agregador, entrega a los artistas del $85 \%$ al 92,5\% de los ingresos brutos y el precio del servicio se basa en una tasa fija anual que empieza desde us\$7 y se incrementa dependiendo del número de canciones y del tipo de servicio escogido ${ }^{24}$.

De esta manera, los agregadores son el conducto mediante el cual artistas o discográficas independientes pueden tomar provecho de los nuevos canales de distribución y recolectar sus regalías, luego del pago de unos costos de intermediación.

\section{Nuevas dificultades para los artistas en el mundo digital}

Como se mencionó, internet no era el escenario preferido de los artistas para poner sus obras a disposición del público, debido a la piratería. Esta situación ha cambiado, ya que se han desarrollado nuevos modelos de negocio legales dándoles una oportunidad incluso a músicos y discográficas independientes de dar a conocer su repertorio a escala mundial. No obstante, dicho escenario no es perfecto. Existen nuevas problemáticas que deben afrontar los artistas, muchas veces debido a la existencia de los intermediarios, como se explicará adelante. Los artistas independientes colombianos se enfrentan a adversidades adicionales debido a la actual oferta de servicios de intermediación, tal como se describe a continuación.

\section{A. Falta de transparencia acerca de la repartición de regalías y baja remuneración a los artistas}

Los nuevos modelos de negocio de la música en internet son complejos, debido a la pluralidad de transacciones, intervinientes y derechos que confluyen. Primero, las plataformas digitales de música funcionan a través de micropagos que realizan los usuarios por cada una de las transacciones, es decir, por descargar una canción, por escuchar un álbum, entre otros ${ }^{25}$. Segundo, existe un gran número de intervinientes en el sistema; por ejemplo, plataformas digitales, agregadores, usuarios, discográficas, editores, sociedades de gestión colectiva, autores, intérpretes, ejecutantes y productores de fonogramas. Tercero, existe una diversidad

23 EmuBands, Simple Pricing, s. f. Recuperado de https:/www.emubands.com/prices/

24 RecordUnion, Find your Plan, s. f. Recuperado de https://www.recordunion.com/pricing.html

25 Rethink Music. Fair Music (música justa)... cit., 3. 
de derechos a recaudar con destino a diferentes personas; por ejemplo, los derechos de autor destinados a los compositores ${ }^{26}$ y los derechos conexos destinados a los intérpretes, ejecutantes y productores de fonogramas ${ }^{27}$.

Dada la complejidad del escenario, este requeriría de un manejo transparente; desafortunadamente, no ha existido transparencia en el sistema acerca de los ingresos que obtiene cada una de las partes ${ }^{28}$. Lo anterior ha generado que sea una tarea difícil determinar cuánto ganan los músicos por sus composiciones ${ }^{29}$ e interpretaciones utilizadas en internet, y muchas veces, aunque se sabe que deben existir pagos, es difícil determinar dónde está el dinero o quién debe pagar. Esto ha desembocado en diversas críticas por parte de los artistas al sistema, debido a lo bajo de sus pagos, como se describirá adelante.

La falta de transparencia se refleja en diversos aspectos del proceso; por ejemplo, los artistas reciben generalmente informes poco claros acerca de los ingresos que se han obtenido por la utilización de sus obras en streaming o en descargas. Esta incertidumbre beneficia a los intermediarios, que pueden ser agregadores o discográficas dependiendo del caso, y también genera que grandes pagos se hagan a las partes equivocadas ${ }^{30}$. Igualmente, esta falta de claridad dificulta cualquier posibilidad de reclamación.

Además, la falta de transparencia se ve empeorada debido a la serie de acuerdos privados y confidenciales que puedan existir entre los artistas y sus discográficas o editores, y a su vez entre las discográficas y las plataformas digitales. En el caso de artistas representados por discográficas, son inicialmente estas las que reciben el dinero por la utilización de las obras y pagan a sus representados de acuerdo con sus contratos, sin que los artistas tengan conocimiento de cuánto están recibiendo originalmente dichas discográficas ${ }^{3}$. De igual manera, los contratos celebrados entre productores de fonogramas y artistas no son actualizados para responder a la realidad del entorno digital, lo que beneficia los ingresos del productor $^{32}$.

26 Ver el Convenio de Berna para la Protección de Obras Literarias y Artísticas (I97I), art. 2, acerca de la protección de composiciones musicales como una obra de derechos de autor; por tanto, las regalías sobre la explotación de dicha obra corresponden al autor de la composición.

27 Ver la Convención de Roma sobre la Protección de Artistas, Intérpretes o Ejecutantes los Productores de Fonogramas y los Organismos de Radiodifusión (octubre 26 de I96r), que establece los derechos que se les deben reconocer a los artistas, intérpretes y ejecutantes y a los productores de fonogramas. Recuperado de http://www.wipo.int/wipolex/es/treaties/text. jsp?file_id=289796

28 омpi, Comité Permanente de Derechos de Autor y Derechos Conexos. Propuesta de análisis de los derechos de autor en el entorno Digital, 20 I 5, 6; Rethink Music. Fair Music (música justa)..., cit., 3.

29 Rethink Music. Fair Music (música justa)..., cit., 7.

30 Ibid., I 2 .

3 I Ibid., 7 .

32 ompi, Comité Permanente de Derechos de Autor y Derechos Conexos, Propuesta de análisis de los derechos de autor en el entorno digital, cit., 6. 
Por otro lado, los artistas, cuando buscan distribuir su música a través de plataformas digitales, no tienen claridad del porcentaje de ingresos que van a recibir, ya sea porque la información no está disponible o porque los pagos se basan en el desarrollo de fórmulas complejas. Así, las regalías de Spotify se basan en una fórmula difícil, casi imposible de verificar por falta de acceso a datos, tales como el total de ingresos de Spotify, total de streamings, entre otros ${ }^{33}$.

De igual manera, intentar saber cuánto paga YouTube por la monetización de las canciones es una tarea prácticamente imposible. El pago de YouTube a los artistas se basa en un porcentaje percibido por la publicidad puesta para sus obras; no obstante, no toda la publicidad se paga de la misma manera; por ejemplo, algunas propagandas se pagan con base en el número de vistas, mientras que otras se pagan según el número de accesos. Igualmente, el precio de la publicidad variará dependiendo de la localización geográfica y del idioma. Así mismo, hay ingresos causados en YouTube que serán compartidos con la persona que realizó el video. Finalmente, se debe aclarar que YouTube es el encargado de decidir cuál publicidad se pone en el video ${ }^{34}$. De esta forma, calcular el monto de ingresos se vuelve una tarea compleja.

Las circunstancias descritas anteriormente generan que, a pesar de que existe un crecimiento en el mercado de los servicios de streaming, y que existe un crecimiento en la industria de la música, los artistas ven una disminución en sus ingresos respecto de ventas análogas ${ }^{35}$, y que esto sea difícil de entender o justificar, debido a las cuentas oscuras. Artistas como David Byrne, Taylor Swift, Radiohead, Bob Dylan, Metallica y Pink Floyd, entre otros, se han pronunciado acerca de los bajos pagos que reciben los artistas de plataformas como Spotify. Muchos de ellos han decidido incluso retirar toda su música de la plataforma, bajo el entendido que dicho modelo de negocio no valora el trabajo de los artistas, debido a la escasa remuneración que estos reciben ${ }^{36}$.

Infortunadamente, como afirma David Byrne, estos bajos pagos perjudican principalmente a artistas que no tienen otras fuentes de ingresos, como mer-

33 Pérez, M., ¿Cuánto dinero paga Spotify a los artistas? Todos los detalles. Tuapplemundo, marzo 8 de 2015. Recuperado de https://www.tuapplemundo.com/cuanto-dinero-paga-spotify-a-losartistas-todos-los-detalles/

34 Industria Musical, “¿Cómo ganan dinero los músicos en YouTube?”, agosto 22 de 2013 . Recuperado de http://industriamusical.es/como-ganan-dinero-los-musicos-en-youtube/

35 Gutiérrez, V., "Adiós al formato físico y la compra de música digital”, junio ig de 20i6. Recuperado de http://eleconomista.com.mx/entretenimiento/2016/o6/I9/adios-formato-fisicocompra-musica-digital

36 Byrne, D., "The Internet will suck all creative Content out of the World", The Guardian, octubre i I de 20I3. Recuperado de https://www.theguardian.com/music/20I3/oct/I I/davidbyrne-internet-content-world; Milenio Digital, "Taylor Swift explica por qué dejó Spotify", noviembre 6 de 20I4. Recuperado de http://www.milenio.com/hey/musica/Taylor_Swift-Spotify-I989_Taylor_Swift-Taylor_Swift_deja_Spotify_o_404359965.html 
chandising o presentación; en otras palabras, músicos independientes o que se encuentran en los comienzos de su carrera ${ }^{37}$.

\section{B. Dificultad para saber cuáles regalías se están recolectando: Colombia}

Aunque internet tiene alcance mundial y los límites geográficos no afectan el intercambio de información, lo cierto es que respecto de derechos de autor no existe un sistema internacional de protección. Cada país presenta su propia legislación autoral, que muchas veces varía de una jurisdicción a $\operatorname{otra}^{3}{ }^{8}$, lo que genera dudas sobre el cumplimiento de las diferentes legislaciones nacionales de derechos de autor en negocios de alcance global. De manera usual, acuerdos de licencias globales de repertorios hacen que se imponga una legislación sobre las demás, sin considerar las características propias de la legislación de cada territorio ${ }^{39}$.

Dicha situación se puede observar especialmente en el caso de los derechos conexos en los acuerdos celebrados entre los artistas independientes y los agregadores, donde al parecer cada agregador recolecta y distribuye las regalías conforme a las leyes bajo las cuales se rige dicho intermediario, mas no según las leyes del lugar donde residen los artistas, ejecutantes y productores de fonogramas o las leyes del lugar donde se utiliza la obra, como se describirá adelante. Esta situación puede perjudicar especialmente a los artistas o discográficas independientes nacionales, residentes, o que se utilicen sus interpretaciones en territorios donde todavía no funcionen agregadores locales, como en el caso de Colombia ${ }^{\circ}$.

Las legislaciones alrededor del mundo presentan diferencias acerca del tratamiento de la protección al fonograma y en especial al derecho de remuneración por la comunicación pública de la obra fonograbada, el cual está involucrado en el caso de streaming ${ }^{4 \mathrm{I}}$. Lo anterior se debe a que a escala internacional no existe un modelo uniforme y vinculante sobre su tratamiento. La protección a los intérpretes, ejecutantes y productores de fonogramas se estableció en la

37 Byrne, D. “The Internet will suck...”, cit.

$3^{8}$ Galuszka, P., "Music aggregators and Intermediation of the Digital Music Market", cit., 26I

39 омpi, Comité Permanente de Derechos de Autor y Derechos Conexos, Propuesta de análisis de los derechos de autor en el entorno digital, cit., 6-7.

40 Durante la realización de esta investigación la autora no tuvo conocimiento de ningún agregador constituido en Colombia.

4I Monroy, J. C., Rojas, X., Sáenz, J. y Arias, C., Los derechos de autor y los derechos conexos en la industria de la música, Bogotá, Dirección Nacional de Derechos de Autor, s. f., 33. Recuperado de http://www.derechodeautor.gov.co/documents/ı I 8 I/I I $769 /$ musica.pdf/e32dcreeodfb-465c-82ce-b534dfdi $6 \mathrm{cb} 4$ 
Convención de Roma de I96 $^{\left[4^{22}\right]}$. Dicho tratado establece en el artículo i 2 el derecho de remuneración equitativa en favor de los intérpretes y ejecutantes o productores de fonogramas cuando un fonograma creado con fines comerciales se utilice directamente para la radiodifusión o cualquier comunicación al público. No obstante, dicho artículo, a pesar de ser el punto más importante del tratado, se incorporó de una manera amplia y con poca coercibilidad en orden de acomodar las posiciones de los diversos países contratantes ${ }^{43}$.

El artículo i 2 de la Convención de Roma permite al Estado contratante escoger que en el momento en que un fonograma creado con fines comerciales sea directamente utilizado para la comunicación pública se abone una remuneración equitativa, bien sea a los intérpretes y ejecutantes o a los productores de fonogramas o a ambos. Es decir, el Estado contratante tiene tres opciones de implementación. Adicionalmente, la aplicación de este artículo se hizo facultativa para los Estados miembros, ya que este podía ser objeto de reservas según lo establece el artículo i6 de la misma Convención44. En consecuencia, la amplitud del texto del tratado genera una falta de uniformidad en los sistemas nacionales respecto del derecho de remuneración en caso de la comunicación pública de obras fonograbadas. De igual manera, esta falta de uniformidad también se debe a que la Convención de Roma tiene solamente 92 estados contratantes 45 .

A escala nacional, Estados Unidos es uno de los países con un modelo de protección a los fonogramas, y en especial al derecho de comunicación pública sobre la obra fonograbada, más característico. Estados Unidos no es miembro de la Convención de Roma. La ley estadounidense de derechos de autor, Copyright Act of I 976, en su sección IO2 reconoce protección al fonograma como una obra protegida por el derecho de autor, $4^{6}$ es decir, en Estados Unidos el productor de fonogramas obtiene derechos de autor sobre dicho fonograma, y no derechos conexos como en otras jurisdicciones.

Bajo este entendido, la ley estadounidense le otorga derechos exclusivos al titular del fonograma como aquellos que se tendrían sobre cualquier otra obra protegida por el derecho autoral: reproducción, distribución y transformación 47 No obstante, el derecho de comunicación pública sobre fonogramas fue limi-

Convención de Roma sobre la Protección de Artistas, Intérpretes o Ejecutantes los Productores de Fonogramas, y los Organismos de Radiodifusión, octubre 26 de г96r. Recuperado de http:// www.wipo.int/wipolex/es/treaties/text.jsp?file_id=289796

43 Azaola, J. M., Organización Mundial de la Propiedad Intelectual (ompi). Guía de la Convención de Roma y del Convenio Fonogramas, Ginebra, ompi, i982, 6I-7I. Recuperado de http://www. wipo.int/edocs/pubdocs/es/copyright/6 I 7/wipo_pub_6 I 7.pdf

Ibid., 6 I-7 I.

Organización Mundial de la Propiedad Intelectual (омPI), Tratados administrados por la OMPI, s. f. Recuperado de http://www.wipo.int/treaties/es/ShowResults.jsp?lang=es\&treaty_id=I 7

46 Copyright Act of 1976, Section Io2 (7) (U. S.).

47 Copyright Act of 1976 , Section 106 (U. S.). 
tado. La Copyright Act de 1976 de manera expresa reconoce como derecho exclusivo sobre el fonograma el derecho de comunicación al público por una transmisión de audio digital $4^{8}$, es decir, aquella que se da por internet o por radio satelital, pero no reconoce un derecho de comunicación pública por radiodifusión terrestre ${ }^{49}$. La diferenciación se realizó porque se entendía que los servicios tradicionales de radio no generaban una amenaza para la industria, como sí lo podría hacer una transmisión de audio digital ${ }^{\circ}$.

Respecto de las comunicaciones públicas en transmisión de audio digital, el Congreso estadounidense diferenció entre servicios no interactivos e interactivos. Según la Copyright Act, los servicios interactivos son aquellos donde el usuario puede recibir la transmisión de un programa creado específicamente para él, o aquellos en los cuales el usuario puede escoger la transmisión de un fonograma específico ${ }^{5}$. Los servicios no interactivos se han entendido como aquellos en los cuales el usuario no tiene la capacidad de alterar o escoger el programa musical ${ }^{52}$. En cuanto al tratamiento jurídico, las transmisiones digitales no interactivas se establecieron bajo un sistema de licencias obligatorias ${ }^{53}$, al igual que las copias efímeras que se pueden realizar durante el proceso automático de la transmisión digital54. Dichas regalías son recaudadas por una entidad llamada Soundexchange ${ }^{55}$. Respecto de los servicios interactivos, las licencias se otorgan bajo negociación individual con el titular de derechos del fonograma ${ }^{56}$.

Ahora, referido a las regalías, bajo el sistema estadounidense se estableció que el derecho a recibir regalías por el uso del fonograma, incluyendo comunicación pública, lo tiene el productor del fonograma, y este les pagará a los artistas y a los músicos acompañantes de acuerdo con sus términos contractuales ${ }^{57}$. No obstante, en el caso de la comunicación pública por transmisión digital no interactiva, los intérpretes y ejecutantes tendrán el derecho de percibir el $45 \%$ de las regalías recaudadas ${ }^{8}$.

48 Copyright Act of 1976 , Section 106 (6) (U. S.).

49 Copyright Act of 1976 , Section I I4 (d) (I) (U. S.).

50 United States Copyright Office, Copyright and the Music Marketplace, 2016, 44. Recuperado de https://www.copyright.gov/policy/musiclicensingstudy/copyright-and-the-music-marketplace.pdf

5 I Copyright Act of I976, Section I I4 (j) (7) (U. S.).

52 Craft, K., "The Webcasting Music Revolution is Ready to Begin, as Soon as we Figure Out the Copyright Law: The Story of the Music Industry At War with Itself", Hastings Communication and Entertainment Law Fournal, 24, 2002, I 5 .

53 Copyright Act of 1976 , Section I I 4.

54 Copyright Act of I976, Section I I 2.

55 United States Copyright Office, Copyright and the Music Marketplace, cit., 47.

56 Copyright Act of 1976 , Section I I 4 (d) (3) (C).

57 Copyright Act of I976, Section I I 4 (g) (I).

$5^{8}$ Copyright Act of 1976 , Section I I 4 (g) (2). 
Lo anterior significa respecto de los artistas, intérpretes y ejecutantes que en Estados Unidos sus derechos de percibir remuneración sobre la comunicación pública de sus interpretaciones son muy limitados, ya que por mandato legal solo tendrán derecho a percibir una remuneración en caso de comunicación pública por transmisión digital no interactiva, como las que se dan en plataformas como Pandora o Spotify gratuito, es decir, aquellos servicios en que el usuario elige un artista o una categoría música o ritmo musical, y la plataforma ofrece una serie de canciones sin que el usuario las haya elegido directamente. Dicha remuneración será recaudada y pagada por Soundexchange directamente a los artistas. En los demás casos de comunicación pública a los artistas, intérpretes y ejecutantes no se les fijó un porcentaje de regalías a recaudar por ley; en este caso recibirán solo la suma o porcentaje negociado contractualmente con el productor de fonogramas.

El escenario estadounidense se puede contrastar con el sistema colombiano. Colombia es signatario de la Convención de Roma desde el i 7 de junio de I97 $^{[59]}$. La ley de derechos de autor colombiana les otorga a los artistas, intérpretes y ejecutantes una serie de derechos patrimoniales y morales ${ }^{60}$ de- $^{-}$ nominados derechos conexos de manera independiente a los derechos de autor que tendría, por ejemplo, el compositor de la obra musical. Entre los derechos patrimoniales se encuentra el derecho de remuneración ${ }^{6 \mathrm{I}}$.

Dicho derecho está establecido en el artículo I73 de la Ley 23 de I982, modificado por el artículo 69 de la Ley 44 de I993; como su nombre lo dice, consiste en el derecho que tienen los artistas, intérpretes y ejecutantes de recibir una remuneración equitativa por la radiodifusión directa de un fonograma hecho con fines comerciales o su reproducción o para cualquier otra forma de comunicación al público. No obstante, dicho derecho no autoriza a prohibir o a autorizar el uso de dichos fonogramas. El derecho de remuneración también se establece en favor del productor del fonograma y se deberá partir en proporciones iguales ${ }^{62}$. Por lo tanto, el Estado colombiano optó por escoger la remuneración equitativa a los artistas y los productores, como lo permite el artículo I 2 de la Convención de Roma.

Debido al derecho de remuneración, y en contraposición a la ley estadounidense, cada vez que un fonograma o su reproducción se utilicen en una estación de radio deberá existir una retribución económica para los artistas, intérpretes y ejecutantes y el productor del fonograma ${ }^{6}$.

60 Ley 23 de I982, arts. I66-I 73; Decisión Andina 35 I de I993, arts. 34-35.

6I Ley 23 de I982, art. I73.

62 Ley 23 de i982, art. I73.

63 Dirección Nacional de Derechos de Autor, Generalidades, derechos conexos, organismos de radiodifusión, s. f). Recuperado de http://200.91.225.I28/Intranei/desarrollo/CONCEPTOSWEB/ arch_conceptos/I-20 I 2-66647.pdf 
De igual manera, la ley colombiana otorga el derecho de remuneración para "cualquier otra forma de comunicación al público"; dentro de este concepto entra la puesta a disposición de fonogramas. La puesta a disposición hace referencia a cuando el público puede acceder a la obra desde el lugar y momento que cada uno desee ${ }^{64}$, es decir, como en el caso de una obra a la que pueda accederse en internet. En la legislación colombiana se ha entendido que la puesta a disposición es una comunicación al público ${ }^{65}$. Por lo tanto, cuando un fonograma es comunicado a través de internet, los artistas e intérpretes tienen derecho a una remuneración.

Igualmente, teniendo en cuenta que el artículo I 73 establece el derecho de remuneración para un acto de comunicación pública descrito de manera amplia, dicho derecho de remuneración sobre la puesta a disposición cobijará tanto los servicios interactivos como no interactivos, sin lugar a diferenciación alguna; en consecuencia, a diferencia de la legislación estadounidense, bajo la legislación colombiana los artistas tiene derecho a recibir una remuneración por cualquier tipo de streaming de sus interpretaciones que se dé en internet.

Ahora, la anterior diferenciación cobra importancia en el momento en que un artista colombiano, que resida en Colombia y cuyas obras se explotan en Colombia, decide contratar a un agregador estadounidense (ya que no hay agregadores colombianos) para que distribuya sus obras en las plataformas digitales y recaude por dichos usos las regalías correspondientes, ya que se crearía una dificultad para saber cuáles derechos se están recaudando y posiblemente recibirá menores regalías que a las que tiene derecho bajo la legislación colombiana.

Para ejemplificar la situación anterior se supondrá que un artista independiente que está en los inicios de su carrera lanza su primer disco por sus propios medios; es decir, para el derecho de autor y los derechos conexos, dicho artista también será el productor de fonograma. De igual manera, se supondrá que sus obras se oyen y descargan en territorio colombiano y que decide contratar a un agregador estadounidense como TuneCore para distribuir sus obras en internet y recolectar regalías. El artista no es miembro de una sociedad de gestión colectiva.

Según los servicios y los términos y condiciones abiertos al público por TuneCore, se puede determinar que este recolecta regalías con base en la legislación de Estados Unidos bajo la cual se constituyó como empresa. Dado este panorama se tiene que:

Si el artista anteriormente mencionado decide contratar la licencia de distribución de TuneCore, dicho agregador se va a encargar de recolectar los master rights, es decir, los derechos que le pertenecen al productor por la explotación

64 Tratado de la ompi sobre Interpretación o Ejecución de Fonogramas, I996, arts. io, I4.

65 Monroy, J. C., Rojas, X., Sáenz, J. y Arias, C., Los derechos de autor y los derechos conexos en la industria de la música, cit., 33 . 
del fonograma ${ }^{66}$. De esta forma, dentro de las regalías recaudadas, TuneCore recaudará los ingresos por streaming de servicios interactivos de las obras en plataformas como YouTube o Spotify. Dichos ingresos son de especial complejidad, ya que presentan un componente por reproducción temporal y otro por comunicación pública de la obra por ser los dos derechos involucrados durante el streaming de una obra ${ }^{67}$. Al ser TuneCore un agregador estadounidense, ambos componentes serán pagados al productor de fonogramas de acuerdo con la legislación norteamericana. Lo anterior genera un problema, ya que según la legislación colombiana el componente por comunicación pública debería pagarse en partes iguales a los intérpretes, artistas y ejecutantes y a los productores de fonogramas. Sin embargo, en el escenario descrito puede que este pago no afecte al artista, ya que estamos bajo el entendido que el artista es ambos: intérprete y productor de fonogramas.

Por otro lado, TuneCore, al trabajar según la legislación estadounidense, no recolectará para el artista los derechos generados por la comunicación pública en plataformas digitales con servicios no interactivos, ya que bajo la ley estadounidense dichos derechos son recaudados por Soundexchange ${ }^{68}$. De esta manera, el agregador estaría recolectando menos regalías que las que el artista debería recibir según la ley colombiana.

Dicha situación se vuelve más preocupante si se tiene en cuenta, primero, que el contrato entre un agregador y un artista implica exclusividad para el segundo y, por lo tanto, el artista no podría firmar con otro agregador que se encargue de recolectar esos derechos. Segundo, si el artista en cuestión no está registrado en SoundExchange -y no sería ilógico presumir que posiblemente no lo esté pensando que su agregador recolecta todos los dineros que se le deben-, la pregunta es: ¿dónde está o quién tiene el dinero que se le debe a dicho artista por los conceptos dejados de recolectar?

El anterior escenario no sucede únicamente con agregadores estadounidenses, sino con los demás agregadores constituidos alrededor del mundo. El mencionado ejemplo demuestra que los artistas en general y en especial los colombianos que buscan tomar provecho de las nuevas oportunidades que brinda el mundo digital se enfrentan a una situación donde, debido a la carencia de agregadores locales y a la existencia de contratos que imponen legislaciones au-

66 Para ver las dos licencias con TuneCore: TuneCore, "What is the difference between Music Distribution and Publishing Administration? Do I need Publishing Administration", cit.

67 TuneCore, "Does TuneCore automatically pay out mechanical royalties to rights/collection societies?", TuneCore, julio 7, 20I6. Recuperado de http://help.tunecore.com/app/ answers/detail/a_id/IO3/ /does-tunecore-automatically-pay-out-mechanical-royalties-torights $\% 2$ Fcollection

68 TuneCore, "What is SoundExchange and how do I collect money from them that is not paid to TuneCore?", abril 26, 20 I6. Recuperado de http://help.tunecore.com/app/answers/detail/a_ $\mathrm{id} / 8 \mathrm{I} / \mathrm{related} / \mathrm{I}$ 
torales extranjeras sobre la nacional, se genera un escenario complejo en el cual es difícil entender y distinguir cuáles derechos se están recaudando, ya que sería necesario estudiar la legislación de derechos de autor del país donde se constituyó el agregador para lograr entender y determinar cuáles derechos se estaría recolectando.

De igual manera, y debido a lo anterior, incluso se podría afirmar que muy probablemente los artistas colombianos estén recibiendo menos regalías que aquellas a las cuales tienen derecho bajo la legislación nacional, tal como se explicó en el ejemplo.

\section{La obligación de someterse a jurisdicciones extranjeras}

Una dificultad adicional para los artistas colombianos es la obligación de someterse a jurisdicciones extranjeras, muchas veces con lenguajes foráneos, para resolver cualquier controversia con su intermediario. Los agregadores buscan regirse muchas veces por las normas del país donde se constituyen y esto incluye fijar como jurisdicción pasiva y leyes a aplicar aquellas del lugar donde se encuentra su domicilio principal.

Dos ejemplos son los agregadores TuneCore o Bquate, de los pocos, por no decir únicos, agregadores de la región. El primero establece en sus términos y condiciones:

(e) The Terms of Service shall be governed by and interpreted in accordance with the laws of the State of New York excluding that body of law pertaining to the conflict of laws. Any legal action or proceeding arising under the Terms of Service shall be brought exclusively in courts located in the State and County of New York, and the parties hereby irrevocably consent to the personal jurisdiction and venue therein $[\ldots]^{69}$.

El segundo, siguiendo el mismo panorama, establece:

Los presentes Términos y Condiciones Generales, así como los Términos y Condiciones Particulares se rigen, en todo lo no dispuesto en el presente documento, por las leyes aplicables de la República del Perú.

69 TuneCore, "Terms and Conditions", mayo I, 2013. Recuperado de http://www.tunecore.com/ terms 
Bquate y el Usuario renuncian expresamente a cualquier otro fuero y/o jurisdicción, y acuerdan someterse a la jurisdicción de los Juzgados y Tribunales del Distrito Judicial del Cercado de Lima, en la República del Perú7º.

Aunque escoger la ley que regirá un contrato internacional y los tribunales que conocerán del asunto permite brindar seguridad jurídica preventiva ${ }^{71}$, esta circunstancia genera dificultades en el presente escenario, donde la parte débil, es decir, el artista, prácticamente acepta el derecho conflictual escogido por el agregador al contratar básicamente mediante un contrato de adhesión. La anterior dificultad surge en el supuesto que emprender una reclamación sería más gravoso para los artistas colombianos, ya que no solo significa someterse a los gastos que implican un proceso judicial, sino, peor aún, a las dificultades de hacerlo en otro país, bajo otras leyes y en la mayoría de los casos en otro idioma y, por lo tanto, puede convertirse en una barrera para hacer exigibles sus derechos. Es decir, en dichos contratos el ejercicio de la autonomía conflictual no busca satisfacer los intereses específicos de ambas partes contratantes, como debiese ${ }^{72}$, sino los intereses de la parte más fuerte.

Lo anterior es más preocupante si se tienen en cuenta las demás barreras que afectan a los artistas en el modelo de negocio de la industria musical en internet, como la falta de transparencia y los bajos pagos. Ya que en el primer caso, aun si dicho obstáculo fuera superado y el artista tuviera acceso a información privilegiada que le permitiera deducir que existe un problema en sus pagos, podría dudar en acudir a la justicia si se tienen en cuenta todas las cargas que esto significa. De igual manera, en el segundo escenario, teniendo en cuenta que los pagos que han recibido los artistas se caracterizan por ser bajos, estos podrían decidir renunciar a sus derechos, debido a que las altas costas pueden superar la cuantía de sus pretensiones.

De tal forma, se estaría frente a un escenario donde se favorecería a los intermediarios en lugar de proteger al artista, ya que la falta de reclamaciones debido a las renuncias de los artistas a perseguir sus derechos por las cargas impuestas tendría como beneficiarios a dichos intermediarios, quienes asegurarían los dineros sujetos a controversias.

70 Bquate, "Términos y condiciones", diciembre de 2015. Recuperado de https://www.bquate. com/terminos-y-condiciones

7 I Oviedo, J. "Ley aplicable a los contratos internacionales", Revista Colombiana de Derecho Internacional, 2 I, 2OI 2, I 23.

72 Guerrero, M. "Legislación aplicable a las contratos internacionales de transferencia de tecnología”, La Propiedad Inmaterial, I 4, 20 Iо. 


\section{Conclusión}

Internet brinda nuevas oportunidades de negocios legales para la industria musical y en especial para artistas o discográficas independientes. Esto se debe a la gran proliferación y crecimiento de plataformas digitales de música que ofrecen diferentes modelos de negocios, como streaming online o descargas digitales, ya sea tras el pago de una subscripción o financiados por publicidad.

Dichos servicios permiten a los artistas darse a conocer, distribuir, comercializar y obtener regalías, lo cual es una gran oportunidad para los artistas que están en el comienzo de sus carreras o que no desean firmar un contrato con un gran sello discográfico.

No obstante, contrario a las estadísticas, los artistas no han podido beneficiarse de la manera esperada de dichas oportunidades. A pesar del crecimiento de la industria digital, varios artistas a nivel mundial han denunciado las bajas regalías que reciben de dichos servicios, muchas veces inexplicables, debido a la falta de transparencia en las transacciones. Por otro lado, y respecto de los artistas colombianos, especialmente los independientes, se observa que sufren otras adversidades al querer utilizar estas nuevas herramientas que brinda la industria de la música digital, en el sentido que se enfrentan a una situación donde, por la carencia de agregadores locales y por la existencia de contratos que imponen legislaciones autorales extranjeras sobre la nacional, se genera un escenario complejo en el cual es difícil entender y distinguir cuáles derechos se están recaudando y donde muy probablemente estén recibiendo menos regalías que aquellas a las cuales tienen derecho según la legislación nacional. De igual manera, cualquier posible reclamación puede verse truncada debido a la obligación que tienen dichos artistas de someterse a jurisdicciones extranjeras.

Por lo tanto, a pesar que inicialmente pareciera estar cambiando la situación de los artistas musicales respecto del uso de internet como canal de explotación de sus obras, en realidad han surgido nuevas problemáticas que afectan a sus intereses, lo que los deja de nuevo como la parte débil del mercado musical.

\section{Bibliografía}

Azaola. J. M., Organización Mundial de la Propiedad Intelectual (омPI), Guía de la Convención de Roma y del Convenio Fonogramas, Ginebra, oмpI, I982, 6I7r. Recuperado de http://www.wipo.int/edocs/pubdocs/es/copyright/6r $7 /$ wipo_pub_6r 7.pdf

Balaban, D. "The Battle of the Music Industry: the Distribution of Audio and Video Works via the Internet, Music and More", Fordham Intellectual Property Media and Entertainment Law Fournal, I2, 2002. 
Blanca. "Justin Bieber. Biografía completa”, Prensa corazón (s. f.). Recuperado de http://laprensa.peru.com/espectaculos/noticia-justin-bieber-biografia-539 I 5

Bquate. "Términos y condiciones", diciembre de 20 I 5. Recuperado de https:// www.bquate.com/terminos-y-condiciones

Byrne, D. "The Internet will suck all creative Content out of the World", The Guardian, octubre II de 20I3. Recuperado de https://www.theguardian.

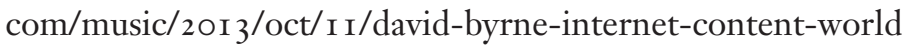

Carrier, M. "No, riaA, It's not the End of the World for Musicians", University of Missouri-Kansas City Law Review, 20 I4. Recuperado de https://papers.ssrn. $\mathrm{com} / \mathrm{sol}_{3} /$ papers.cfm?abstract_id=2602469

“Cómo funciona Content ID", YouTube, s. f. Recuperado de https://support. google.com/youtube/answer/2 797370?hl=es-4I9

“¿Cómo ganan dinero los músicos en YouTube?”, Industria Musical, agosto 22 de 2013 . Recuperado de http://industriamusical.es/como-ganan-dinero-losmusicos-en-youtube/

Congreso de Colombia. Ley 23 de i982, sobre derechos de autor.

Convención de Roma sobre la Protección de Artistas, Intérpretes o Ejecutantes, los Productores de Fonogramas y los Organismos de Radiodifusión (octubre 26 de I96I). Recuperado de http://www.wipo.int/wipolex/es/treaties/text. jsp?file_id=289796

Copyright Act of 1976 (U. S.).

Craft, K., "The Webcasting Music Revolution is Ready to Begin, as Soon as we Figure Out the Copyright Law: The Story of the Music Industry At War with Itself", Hastings Communication and Entertainment Law Fournal, 24, 2002.

Decisión Andina 35 I de I993, Régimen Común sobre Derechos de Autor y Derechos Conexos, Comisión del Acuerdo de Cartagena.

Dirección Nacional de Derechos de Autor. Generalidades, derechos conexos, organismos de radiodifusión, s. f. Recuperado de http://200.91.225.I28/IntraneI/ desarrollo/CONCEPTOSWEB/arch_conceptos/I-20 I 2-66647.pdf 
Donald, A. "Independent Musicians: let Technology Free you From Labels' Yoke", The Associated Press, enero 27, 2004.

EmuBands. Simple Pricing, s. f. Recuperado de https://www.emubands.com/prices/

"Find your Plan", RecordUnion, s. f. Recuperado de https://www.recordunion. com/pricing.html

Galuszka, P. "Music aggregators and Intermediation of the Digital Music Market”, International fournal of Communication, 9, 2015.

Guerrero, M. "Legislación aplicable a los contratos internacionales de transferencia de tecnología”, La Propiedad Inmaterial, i4, 20 Iо.

Gutiérrez, V., "Adiós al formato físico y la compra de música digital”, El Economista, junio I9 de 2016 . Recuperado de http://eleconomista.com.mx/entretenimiento/2016/o6/I9/adios-formato-fisico-compra-musica-digital

Guzmán, J. "La música por Internet le gana la batalla a los discos”, ElTiempo, mayo I 5, 20I6. Recuperado de http://www.eltiempo.com/entretenimiento/musica-y-libros/ventas-de-musica-digital-sobrepasaron-las-de-discos/r 6593353

IFPI. Global Music Report: Music Consumption Exploding Worldwide, 20 I6.

"Justin Bieber: la biografía del cantante canadiense", La Prensa, 29 octubre de 20I 5. Recuperado de http://laprensa.peru.com/espectaculos/noticia-justinbieber-biografia-539 5

"Make more money with music publishing", cDbaby. s. f. Recuperado de https:// members.cdbaby.com/pro.aspx

Mclean. "James Bay interview: 'As a solo artist with a guitar, I can only write so many weepie little bedroom songs", Independent, s. f. Recuperado de http:// www.independent.co.uk/arts-entertainment/music/features/james-bayinterview-as-a-solo-artist-with-a-guitar-i-can-only-write-so-many-weepielittle-bedroom-Io 2 I $453 \cdot \mathrm{html}$

Milenio Digital, “Taylor Swift explica por qué dejó Spotify”, noviembre 6 de 20I4. Recuperado de http://www.milenio.com/hey/musica/Taylor_SwiftSpotify-ı989_Taylor_Swift-Taylor_Swift_deja_Spotify_o_404359965.html 
Monroy, J. C., Rojas, X., Sáenz, J. y Arias, C., Los derechos de autor y los derechos conexos en la industria de la música, Bogotá, Dirección Nacional de Derechos de Autor, s. f. Recuperado de http://www.derechodeautor.gov.co/documents/ı г 8 I/ I I 769 /musica.pdf/e 32 dc г ee-odfb-465 c-82 ce-b534dfd I 6cb4

ompi, Comité Permanente de Derechos de Autor y Derechos Conexos, Propuesta de análisis de los derechos de autor en el entorno digital, 2015.

Oviedo, J., "Ley aplicable a los contratos internacionales", Revista Colombiana de Derecho Internacional, 2 I, 20 I 2.

Pérez, M., “¿Cuánto dinero paga Spotify a los artistas? Todos los detalles”, Tuapplemundo, marzo 8 de 20 5. Recuperado de https://www.tuapplemundo.com/cuanto-dinero-paga-spotify-a-los-artistas-todos-los-detalles/

Rethink Music. Fair Music (música justa): transparencia y flujos monetarios en la industria musical, Boston, Berklee ICE, s.f.

Ríos, W. "Análisis de las sentencias en los casos de Napster, Grokster, Morpheus, Streamcast y Kazaa", La Propiedad Inmaterial, I 2, 2008. Recuperado de https://dialnet.unirioja.es/servlet/articulo? codigo=3 I 35280

Tratado de la ompi sobre Interpretación o Ejecución de Fonogramas, I996.

"Does TuneCore automatically pay out mechanical royalties to rights/collection societies?", TuneCore, julio 7, 2016. Recuperado de http://help.tunecore. com/app/answers/detail/a_id/IO3/ /does-tunecore-automatically-pay-outmechanical-royalties-to-rights $\%_{2}$ Fcollection

"Terms and Conditions", TuneCore, May I, 20I3. Recuperado de http://www. tunecore.com/terms

“TuneCore", s. f. Recuperado de http://www.tunecore.com/

"What is Sound Exchange and how do I collect money from them that is not paid to TuneCore?", TuneCore, abril 26, 20г6. Recuperado de http://help. tunecore.com/app/answers/detail/a_id/8I/related/I

"What is the difference between Music Distribution and Publishing administration? Do I need Publishing Administration?", TuneCore, enero 26, 20 I6. Recuperado de http://pub.help.tunecore.com/app/answers/detail/a_id/255 
United States Copyright Office, Copyright and the Music Marketplace, 20I6, 44. Recuperado de https://www.copyright.gov/policy/musiclicensingstudy/copyright-and-the-music-marketplace.pdf

"Who Needs Record Labels?", Independent, marzo I2, 2010. Recuperado de http://www.independent.co.uk/arts-entertainment/music/features/whoneeds-record-labels- $1920056 . \mathrm{html}$

"Your Music", Spotify, s. f. Recuperado de https://artists.spotify.com/guide 